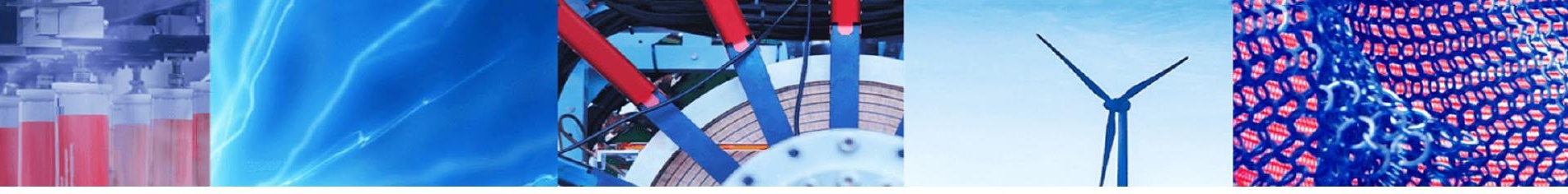

Research Article

\title{
Persistent oscillations in a traffic model with decision-making
}

\author{
Ding-wei Huang ${ }^{1}$ (]
}

Received: 1 August 2019 / Accepted: 11 December 2019 / Published online: 18 December 2019

(c) Springer Nature Switzerland AG 2019

\begin{abstract}
This work explores the nonlinear behaviour in a congestion charge model, which emphasizes the correlation between drivers' response and optimal traffic flow. The model assumes that all drivers follow the same common rules of rational thinking to avoid the congestion. There are two regimes in the model system. In free flow regime, the system has only one branch. In congested regime, the system bifurcates into two branches. Counter-intuitive behaviour of the model is caused by the oscillatory instability between two branches. Persistent oscillations reflect the intrinsic properties of decision-making process. Rational thinking provides limited adaptation capabilities to reach the optimal traffic flow. More past experiences and current options provide only marginal increase in capabilities to adapt to traffic situations. The idealized situations are also discussed.
\end{abstract}

Keywords Congestion charge $\cdot$ Optimal flow · Decision-making · Adaptation · Bifurcation

Mathematics Subject Classification $60 \mathrm{~K} 30 \cdot 90 \mathrm{~B} 20 \cdot 91 \mathrm{~B} 69$

JEL Classification C44 D D79 $\cdot$ R41

\section{Introduction}

Basic and applied sciences have different goals and approaches. Basic sciences focus on the fundamental interactions among the smallest units of nature. The goal is to search a simple description to the behaviour of tiny objects like atoms and electrons. To build an appropriate model, the number of parameters is the smaller the better. In contrast, applied sciences study the effective interactions among big objects like cars and roads. In order to describe the complicate objects, a workable model often involves a large number of parameters. These two approaches are pursued by scholars in different disciplines. One uses few parameters to describe simple things; the other uses many parameters to describe complex things. Recently, a new approach has emerged: using few parameters to describe complex things. The subjects of study are in the realm of applied sciences, while the methodologies of research are in the realm of basic sciences. It is important to find the crucial parameters and to have a wide range of validation. Two examples are traffic physics [1-3] and sociophysics [4-6]. In traffic dynamics, cars moving on the roadway are analogized to particles moving under the influence of a force field. In an idealized situation of all drivers following the rules, traffic regulations can be analogized to the laws of physics within the model system. The relationship between empirical traffic patterns and imposed traffic regulations can be analysed. As the number of parameters is small, a basic model has the capacity to incorporate different phenomena. Thus, highway traffic and local traffic can be discussed in the same framework, which can be further extended to include pedestrian dynamics, granular dynamics, and internet dynamics [7]. To make improvement of traffic conditions, this new

Ding-wei Huang, dwhuang@cycu.edu.tw | 'Department of Physics, Chung Yuan Christian University, Chung-li, Taiwan. 
approach provides a top-down perspective. In contrast, the empirical thumb rules are often from the bottom-up perspective. For example, transport efficiency on complex networks has attracted much attention recently. The structure and dynamics of complex networks have been studied in many different disciplines [8]. A wide range of phase transitions and self-organized critical phenomena have been observed [9]. The self-organized oscillations of traffic flow in street networks were reported [10]. An efficient routing scheme was proposed to find the shortest path in a network without complete information of topology [11]. In some specific cases, the analysis of phase transitions led to the proposal of new strategy to relieve the congestion [12].

The advance of sociophysics illustrates that human behaviour in the social environment can also be analysed from this top-down perspective. Traffic phenomena involve not only the mechanical interactions between cars and roadways, but also the human behaviour of drivers behind the wheels. Besides following the traffic rules, traffic patterns are also influenced by other decision-making behaviour. An optimal traffic model with decision-making has been proposed to show an interplay between human decision and network structure [13, 14]. This work focuses on the typical example of congestion charge scheme [15-17]. Congestion charge is a popular scheme adopted by many modern cities in the hope of easing traffic flow in the gridlocked areas. Based on empirical experiences, congestion charge has been credited with reducing car usage, promoting public transport, cutting pollution emissions, and improving traffic flow $[18,19]$. On the other hand, some contradictory results were also reported [20]. Both public acceptance and drivers' response have been assessed [21-24]. Congestion charge has a wide influence on different facets of a society. This work focuses on the drivers' response in the congestion charge scheme. A model system is used to study the correlation between drivers' response and traffic flow. This study examines whether the drivers' rational response will result in the optimal traffic flow. Unlike other traffic management, the congestion charge scheme does not provide a route assignment for each driver. In stead, the success of congestion charge scheme depends on all drivers' rational decisions in route choice. The model assumes that rational drivers follow traffic rules and decision-making processes. This work further extends previous analysis of decisionmaking in traffic dynamics. This study provides a systematic exploration of the effectiveness of rational decisions in route choice to avoid congestion.

\section{Methodology}

Extensive numerical simulations are performed in a model system. Analytical results are also discussed. The agent-based model simulates a binary decision-making process on a ring-and-hub topology [14]. All agents can move on the ring. Some of the agents have the privilege to access the hub and must decide to travel along the outer ring or through a central hub. The regular route is along the ring. The hub provides as a short cut, but the capacity of the hub is finite. The short cut becomes ineffective when the hub is crowded. Congestion in the hub should be avoided by rational decision makers. The decision-making of each agent is neither predetermined nor random. All agents follow the same rules to make decisions. Yet each agent is allowed the flexibility to learn to adapt to different situations. Agent's behavioural heterogeneity is also recognized.

The system consists of $N$ agents and a ring of $N$ sites, where each site sits an agent. The destination of each agent is randomly assigned. The agents travel along the ring with constant speed. The distance between origin and destination becomes a simple measure of the travelling time or cost, which is denoted as $g$. Travelling along the ring, the cost is the number of sites between origin and destination. Using the hub, the cost reduces to 1 if the hub is uncongested. If the hub is congested, the cost increases to $1+\mathrm{cc}>1$, where $\mathrm{cc}$ denotes the congestion charge. The finite capacity of the hub is $L$. The access to the hub is limited to $\lambda$ agents, whom are chosen randomly. Among these $\lambda$ agents, if more than $L$ agents decide to use the hub, the congestion emerges and the congestion charge $\mathrm{cc}$ is added to the travelling cost. The two most important parameters to influence the traffic dynamics are the number of links $\lambda$ and the congestion charge cc.

The decision-making process is governed by the conventional way of following the best strategy card at hand. Each agent is offered a number of strategy cards. Each strategy card prescribes a deterministic relation between the next choice and the previous ones. The score of each strategy card is evaluated by the success of previous experiences. The number of strategy cards received by each agent is denoted by $s$. The length of memory on each strategy card is denoted by $m$. Each agent has the same number of strategy cards, which are randomly selected from all the possible ones. Yet each agent has different strategies available to use. The score of a strategy card is dynamic. The same strategy card has different scores as time evolves. The same strategy card in different agents' hands has different scores. In the process, each agent must follow his/her highest-score 
strategy to make decision. The decision-making process is controlled by two parameters: the length of memory $m$ and the number of strategy cards $s$.

In the model system, the traffic dynamics has been reduced to a two-route layout: to move along the regular route or to use the short cut. The human decision has been reduced to a selection between these two choices. If only the traffic dynamics were considered, the best choice is to use the short cut when the traffic is uncongested or the congestion charge is low. When the human decision is considered, the congestion is correlated to agents' choices. Raising the congestion charge to suppress the congestion is an expectation depending on all agents' decisions. This model assumes that all agents are reasonable and rational. All agents follow the same common rules to make decisions. Each driver is limited by his own options and experiences. Different options are assigned to each agent initially. Each agent gains different experiences in the process of evolution. With these assumptions, this model explores theoretically the correlation between imposing the congestion charge and suppressing the congestion. In the following, the effects of four parameters $(\lambda, c c, m, s)$ are analysed in details.

\section{Results}

This model emphasizes that the congestion is caused by individual agent's spontaneous decision to take the short cut. The impose of congestion charge provides as a balance force to suppress the use of short cut and thus to relieve the congestion. In an idealized situation, the congestion can be suppressed without a central control of each agent's path assignment. With the prescribed random destination, the maximum cost is $N / 2$ and the average cost is $N / 4$ if all agents travel along the ring. When the hub becomes available, the cost can be expected to reduce; when the congestion charge is imposed, the cost can be expected to increase. When $\lambda$ is small, the congestion is not possible and the congestion charge cc is irrelevant. The cost decreases linearly with the increase of $\lambda$. When $\lambda$ is large, the congestion will emerge if the agents choose to travel through the hub whenever possible. The impose of congestion charge cc provides an incentive for agents to self-organize to avoid the congestion. With reasonable intuition, one would expect that (1) $g$ decreases with an increasing $\lambda$ and (2) $g$ increases with an increasing cc. Typical results are shown in Figs. 1 and 2. In the simulations of $N=101$, data are averaged from 101 to 200 cycles. The first 100 cycles are discarded as transient. The system evolves very quickly towards a steady state. In practice, a stable result can be reached after 10 cycles. An ensemble average over 100 events is further performed to

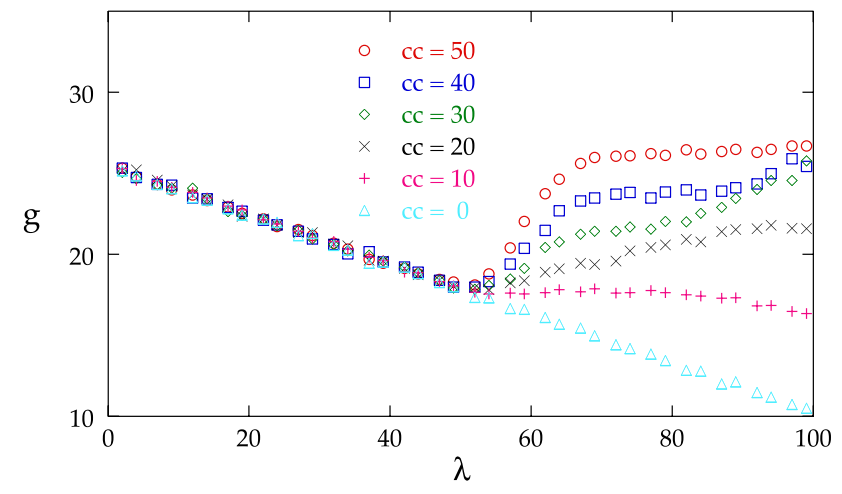

Fig. 1 Typical results of $g(\lambda)$ at $m=2, s=2$, and various values of cc. Other parameters are $N=101$ and $L=40$. Data are averaged from 100 to 200 cycles, with an ensemble average over 100 events

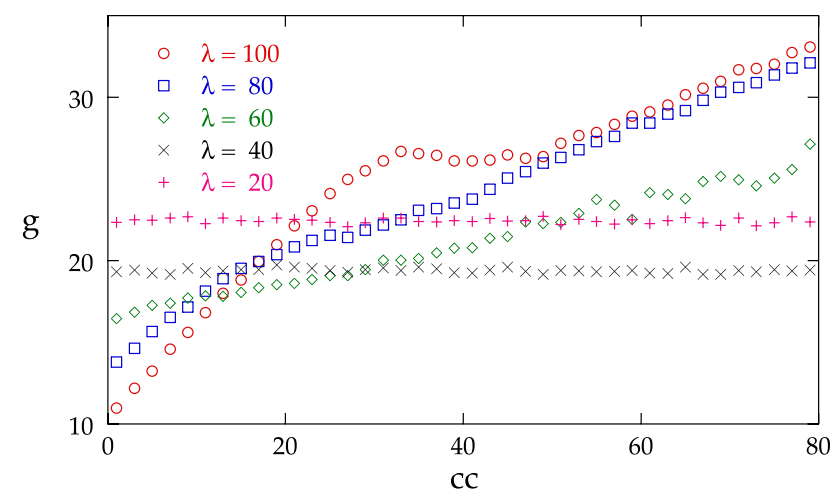

Fig. 2 Typical results of $g(\mathrm{cc})$ at $m=2, s=2$, and various values of $\lambda$. Other parameters are the same as in Fig. 1

have a smooth result. An interesting nonlinear behaviour is observed in the congestion regime. In some parameter range, $g$ increases with an increasing $\lambda$ and/or $g$ decreases with an increasing cc. Such behaviour is counter-intuitive and confusing.

The nonlinear curve $g(\lambda)$ shown in Fig. 1 does not reveal the underlying dynamics of the system. Figure 3 plots the simulation results both in a single cycle (open symbol) and their average over 100 cycles (closed symbol). In the free flow regime, $\lambda<L$, normal fluctuations can be observed. Data points fluctuate around the average value. In the congestion regime, $\lambda>L$, data points divide into two branches. The average value does not represent any real event. The complete evolution of probability profile as a function of both $g$ and $\lambda$ is shown in Fig. 4. When $\lambda<L$, the system presents a single branch. When $\lambda>L$, the system divides into two branches. The nonlinear curve shown in Fig. 1 is the sum over these two branches. The lower branch is independent of both $\mathrm{cc}$ and $\lambda$, while the upper branch moves continuously towards a higher cost as cc increases. The lower branch is the desired result of 


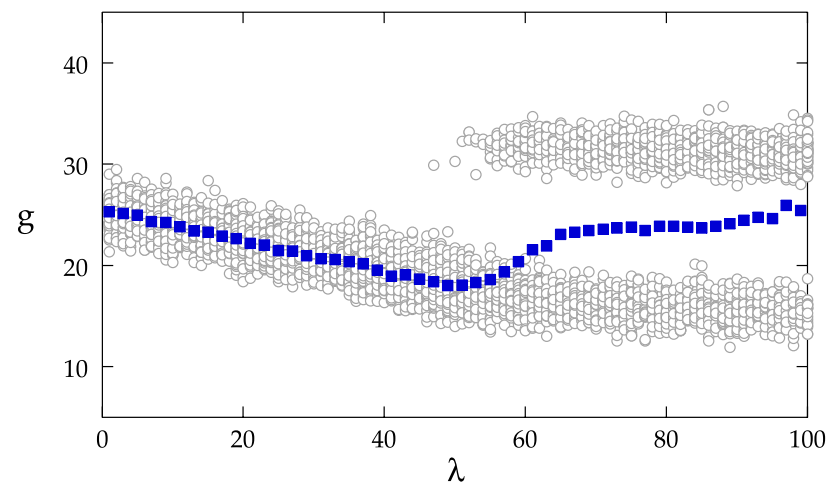

Fig. 3 Typical results of $g(\lambda)$ at $m=2, s=2$, and $c c=40$. Open symbol shows the fluctuations from cycle to cycle; closed symbol shows the averaged results

effective adaptation. In contrast, the upper branch is the result of irrational decisions.

The effects of two other parameters $m$ and $s$ are also studied. These two parameters are correlated. With a given $m$, there are $2^{\left(2^{m}\right)}$ different strategy cards, i.e. $1 \leq s \leq 2^{\left(2^{m}\right)}$. When $s$ is small, the average cost decreases gently with the increase of $\lambda$. The congestion charge becomes effective at a $\lambda$ larger than $L$. As $s$ increases, the average cost presents a steeper slope and the congestion charge onsets at a smaller $\lambda$. Results are shown in Fig. 5 , where the value of $s$ is plotted in a logarithmic scale. The effect of increasing $s$ is not linear, especially when $m$ is large. In the case of $m=3$, the maximum is $s=256$. Yet the result saturates around $s=10$. As $s$ increases, the fast saturation indicates an insufficient adaptation in the evolution processes. The results improve marginally when more strategy cards are available.

\section{Discussions}

In this model, all agents follow the same rules to make decisions. Previous study indicated that the agents self-organize into two groups when the congestion emerges [14]. The long-distance travellers have a higher probability than the short-distance travellers to use the hub, which is a clear demonstration of adaptation. Typical results are shown in Fig. 6 . The congestion charge cc becomes a simple measure of distance to differentiate these two groups. The two groups are not to be identified with the two branches shown in Fig. 3. When the two branches are separated, both branches present the same differentiation of two groups as also shown in Fig. 6. Obviously, formation of the two branches is caused by a different mechanism. Figure 7 plots the same dependence for various values of $s$. In the case of $s=1$, each agent follows his/her only one strategy card to make decision.
Since there is no adaptation, a flat probability at 0.5 is observed, i.e. each agent has equal probability to use or not to use the hub. In the case of $s>1$, each agent can switch strategies to adapt to traffic conditions. A higher probability can be observed for travelling distance larger than cc. As $s$ increases, the difference enlarges between long-distance and short-distance travellers. Increasing $s$ increases the ability to adapt to traffic conditions. Better results can thus be achieved.

The self-organizing into two groups is not the mechanism behind the two branches. The two branches can still be observed in the case of $s=1$, where all the agents have the same probability to use the hub. The branching effect should be attributed to the operation of strategy cards, which imposes strong constraint to the decision-making process. Figure 8 plots the cost $g$ from cycle to cycle, which shows fast oscillations between the two branches. The oscillations can be taken as a manifest of the underlying stochasticity in the decision-making process. Although the decision-making process is deterministic to follow the rules, the operation of strategy cards introduces intrinsic stochasticity into the process.

In this model, the dynamics of decision-making is controlled by two parameters: $m$ and $s$, which represent, respectively, the length of memory in strategy consideration and the number of strategy cards in each agent's hand. A larger $m$ implies each agent has more past experiences to learn from. A larger $s$ implies each agent has more current options to choose from. With naive intuition, increasing the length of memory and/or the number of options will enhance the ability to find a better way to avoid the congestion. However, numerical simulations indicate that the effect is marginal. As the parameter $m$ increases, the strategy variety increases rapidly. The agent's behavioural heterogeneity is controlled by the parameter $s$, where $1 \leq s \leq 2^{\left(2^{m}\right)}$. When $s$ is small, all the agents can be differentiated from one another by the different strategy cards in their hands. As $s$ increases, the heterogeneity diminishes and the agents become more homogeneous. With a small $s$, the ability to adapt to traffic conditions is limited as there are only few options. With a large $s$, the ability is also limited owning to the homogeneity. With more strategy cards at hand, the results improve marginally.

Another indication of insufficient adaptation is the dependence on initial configurations. The score of each strategy card can only be evaluated properly after the first $m$ iterations. In the simulations, random configurations are used as the initial conditions in the first $m$ iterations. In general, the final results are expected to be independent of the initial configurations. In this model, however, the results depend on the initial configurations. The dependence on the initial configurations implies that 
Fig. 4 Probability profiles correspond to the nonlinear curves shown in Fig. 1
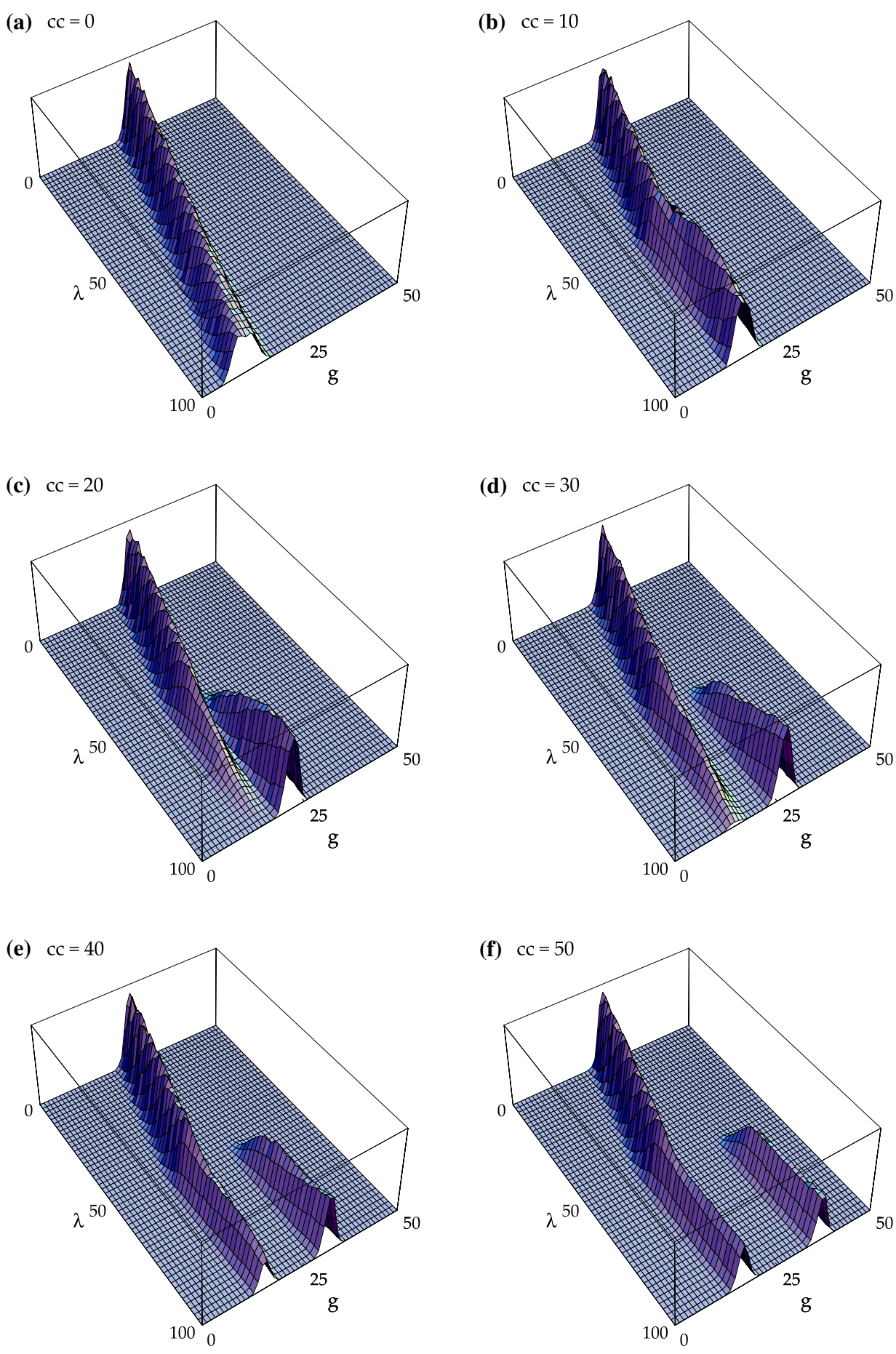

the dynamics cannot provide sufficient adaptation. As $s$ increases, the dependence on the initial configurations diminishes as expected.

In this model, the agents can be divided into two categories in a different way: one has a choice to make and the other has no choice. The typical results of their different distributions are shown in Fig. 9, where the parameters are the same as in Fig. 3. As shown in Fig. 9a, the bifurcation is obvious for those agents who can access to hub and have a choice. In the free flow regime of small $\lambda$, the optimal result is for all the agents to use hub whenever possible. The idealized situation would be $g=1$. However, the operation of strategy cards introduces intrinsic fluctuations to result in $g>1$. In the congestion regime of large $\lambda$, the congestion charge becomes inevitable and the oscillations emerge. With a fixed $N$, the number of make-decision 

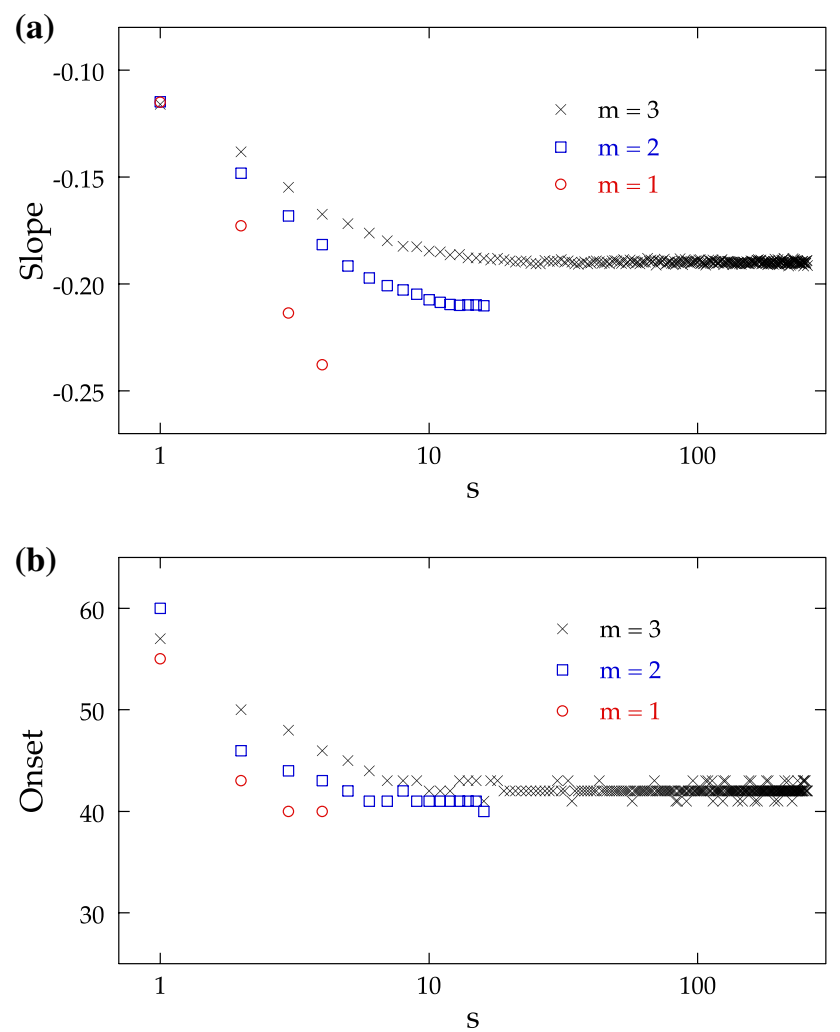

Fig. 5 Typical results to show the fast saturation as $s$ increases: a slope of $g(\lambda)$ in the free flow regime; $\mathbf{b}$ onset of $\lambda$ for the congestion

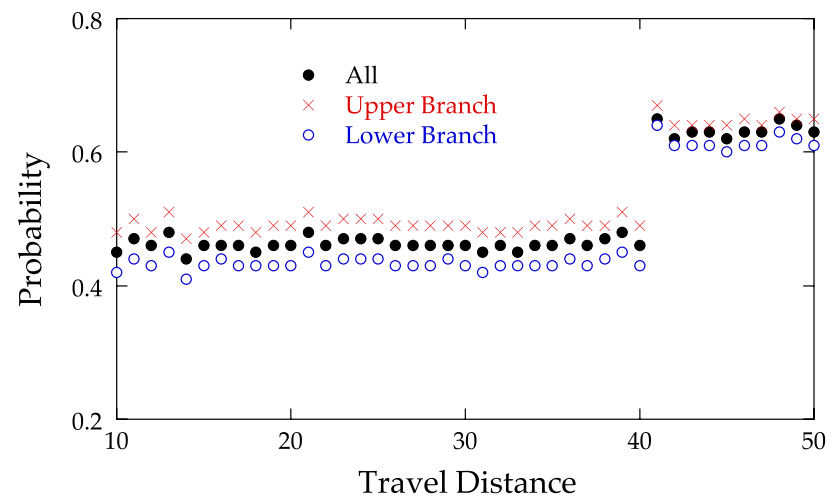

Fig. 6 Travel distance dependence of an agent's probability to use the hub. Parameters are $m=2, s=2, \lambda=80$, and $c c=40$

agents increases with the increase of $\lambda$. As a result, the scattering of data decreases with the increase of $\lambda$. In contrast, Fig. 9b shows the results for those agents who cannot access to hub and have no choice. With a fixed $N$, the number of no-choice agents decreases with the increase of $\lambda$. As a result, the scattering of data increases with the increase of $\lambda$.

Without adaptation, the cost $g$ is a linear function of $\lambda$ and has a sudden jump at $\lambda=L$ as shown in Fig. 10a.

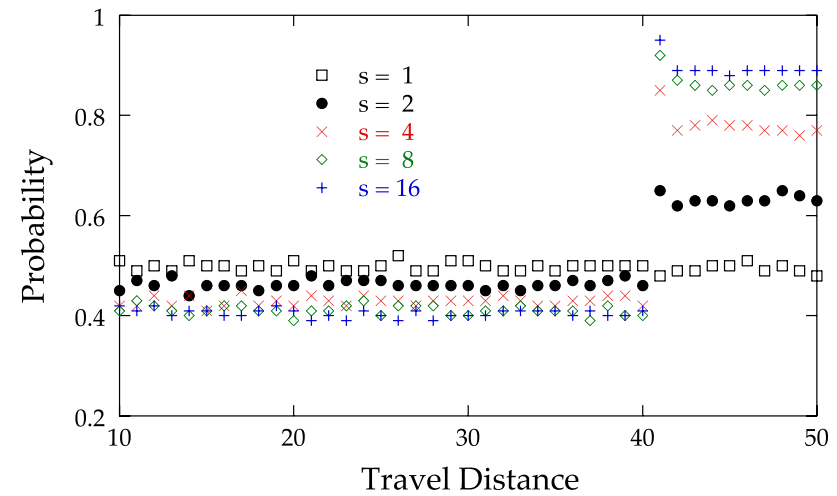

Fig. 7 Travel distance dependence of an agent's probability to use the hub for various $s$. Parameters are $m=2, \lambda=80$, and $c c=40$

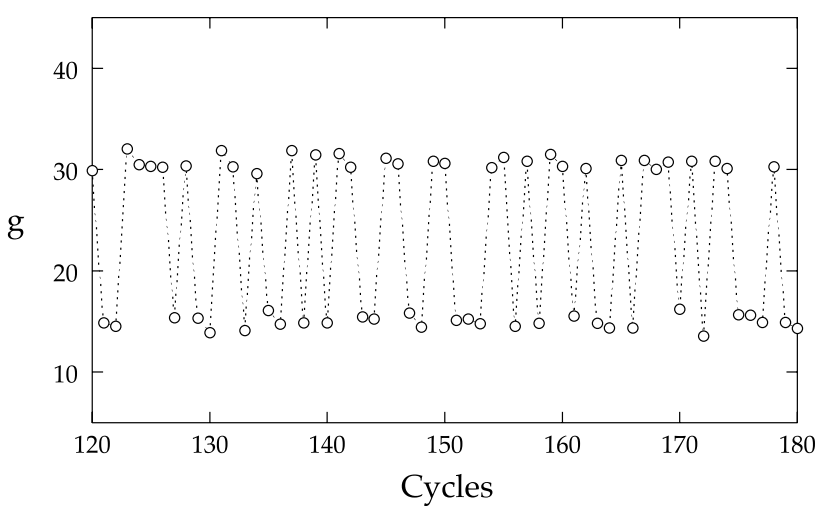

Fig. 8 Typical results of $g(\lambda)$ at $m=2, s=2, \lambda=80$, and $c c=40$

The congestion charge is imposed to discourage the short-distance travellers from using the hub. By rational decision-making, the hub should be reserved for the travelling distance larger than cc. The simulation results are shown in Fig. 10b. The analytical formula can be written as

$g(\lambda)= \begin{cases}\frac{N}{4}-\frac{\lambda}{4}+\frac{\lambda}{N^{\prime}}, & 0<\lambda<L \\ \frac{N}{4}-\frac{\lambda}{4}+\frac{\lambda}{N}-\frac{2 \lambda(\mathrm{cc})}{N^{2}}+\frac{\lambda(\mathrm{cc})^{2}}{N^{2}}, & L<\lambda<\frac{N L}{N-2(\mathrm{ccc})} \\ \frac{N}{4}-\frac{\lambda}{4}+\frac{\lambda}{N}+\frac{\lambda(\mathrm{cc})}{N}-\frac{2 \lambda(\mathrm{cc})}{N^{2}}-\frac{\lambda(\mathrm{cc})^{2}}{N^{2}}, & \frac{N L}{N-2(\mathrm{cc})}<\lambda<N\end{cases}$

when $\mathrm{cc}<(N-L) / 2$, the cost $g(\lambda)$ consists of three line segments. In the simulations, the first transition is abrupt and the second transition is smooth. When $\mathrm{cc}>(N-L) / 2$, only two segments with an abrupt transition are observed. The model simulations shown in Fig. 1 can be understood as a noisy version of this rational decision-making, especially when $s$ is large. The noises include the intrinsic fluctuations and the oscillations between two branches. The idealized situation to avoid the congestion is shown in Fig. 10c, where the hub is used by the long-distance 

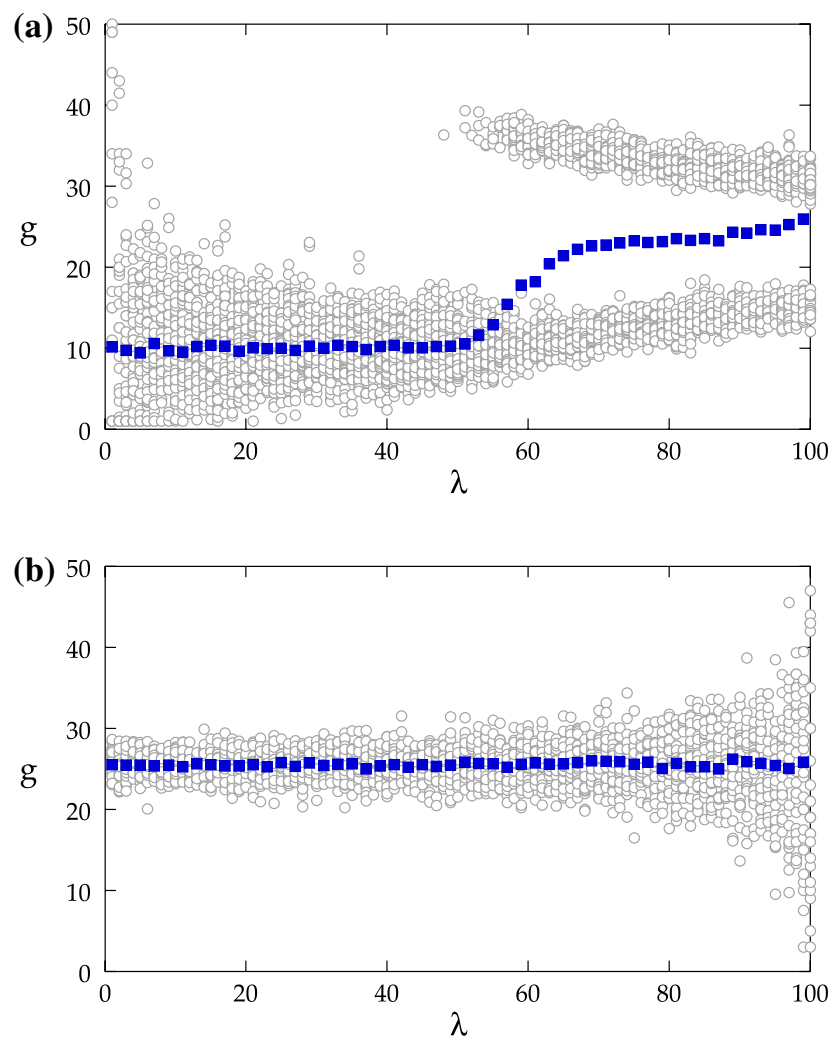

Fig. 9 Typical results of $g(\lambda)$ with two different kinds of agents: a agents can access to hub; $\mathbf{b}$ agents cannot access to hub. Parameters are the same as in Fig. 3

travellers and the number is limited to $L$. The analytical expression for this continuous curve can be obtained as

$g(\lambda)= \begin{cases}\frac{N}{4}-\frac{\lambda}{4}+\frac{\lambda}{N^{\prime}}, & \lambda<L \\ \frac{N}{4}-\frac{L}{2}+\frac{L^{2}}{4 \lambda}+\frac{L}{N^{\prime}}, & \lambda>L\end{cases}$

As congestion will not emerge in this idealized situation, the congestion charge becomes irrelevant. The model simulations are quite far away from this idealized situation. In this model, the decision-making process is controlled by the operation of strategy cards. Each agent is instructed to follow the best strategy card in his/her hands. The results indicate that conflict of interest cannot be resolved in the congestion regime.

\section{Conclusion}

This study reports the nonlinear behaviour of optimal traffic flow in a model system of congestion charge operation. With conventional wisdom, congestion charge scheme seems to be a promising tool to relieve traffic congestion through drivers' rational decisions. The model assumes that all drivers follow the same common rules to make

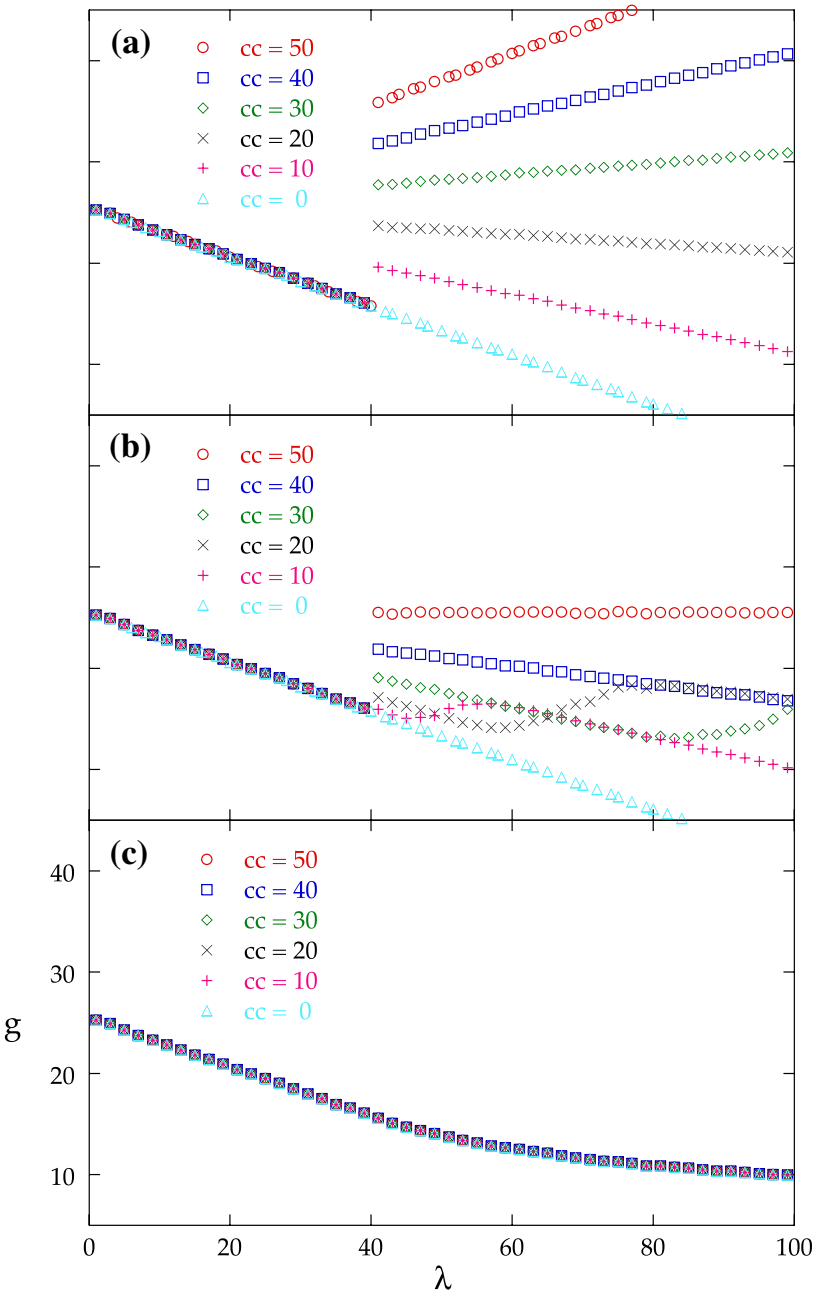

Fig. 10 Analytical results of $g(\lambda)$ : the hub is used by $\mathbf{a}$ all agents, $\mathbf{b}$ agents with travel distance longer than $c c, \mathbf{c} \lambda$ agents with longest travel distance

decisions to avoid the congestion. The best decision leads to the lowest cost $g$. One expects that an increasing link $\lambda$ will result in a decreasing cost $g$, and an increasing congestion charge cc will result in an increasing cost $g$. An increasing link $\lambda$ implies that more agents have access to the short cut. The cost $g$ will decrease if the agents make correct decisions. Similarly, an increasing congestion charge cc will increase the cost $g$. The increment is equal to the congestion charge if the agents cannot adapt. A lesser increment can be observed if the agents are able to adapt their behaviour by making correct decisions. In some parameter regime, counter-intuitive results are observed, i.e. the cost $g$ increases with an increasing link $\lambda$ and/or the cost $g$ decreases with an increasing congestion charge cc. The peculiar behaviour is attributed to the oscillatory instability between two branches. In the free flow regime, the system presents only one branch. In the congested regime, the system bifurcates into two branches. These persistent 
oscillations are originated in the decision-making process. In the daily experience, there are many examples of persistent oscillations. Consider a group of people making independent decisions. Some choose one option, while others choose a different option. If the outcome is not satisfied, people switch their choices. The persistent oscillations indicate a frustrated system in the congestion regime. Incorporating more experiences and more choice options has limited effect to remedy the frustration of congestion. In the model system, the best strategy is evaluated from the perspective of personal interest. The conflict of interest cannot be resolved in the congestion regime. It would be interesting to further explore if the introduction of public welfare would lead to a more satisfied result.

Funding This work was funded by MOST-104-2112-M-033-001.

\section{Compliance with ethical standards}

Conflict of interest The corresponding author states that there is no conflict of interest.

\section{References}

1. Nagatani T (2002) The physics of traffic jams. Rep Prog Phys 65:1331

2. Kerner BS (2004) The physics of traffic: empirical freeway pattern features, engineering applications, and theory. Springer, New York

3. Maerivoet S, De Moor B (2005) Cellular automata models of road traffic. Phys Rep 419:1

4. Chakrabarti BK, Chakraborti A, Chatterjee A (eds) (2006) Econophysics and sociophysics: trends and perspectives. Wiley, Hoboken

5. Castellano C, Fortunato S, Loreto V (2009) Statistical physics of social dynamics. Rev Mod Phys 81:591

6. Galam S (2012) Sociophysics: a physicist's modeling of psychopolitical phenomena. Springer, New York

7. Hamdar SH (ed) (2019) For example, see Traffic and Granular flow'17. Springer, New York

8. Boccaletti S, Latora V, Moreno Y, Chavez M, Hwang DU (2006) Complex networks: structure and dynamics. Phys Rep 424:175
9. Dorogovtsev SN, Goltsev AV, Mendes JFF (2008) Critical phenomena in complex networks. Rev Mod Phys 80:1275

10. Helbing D, Siegmeier J, Lämmer S (2007) Self-organized network flows. Netw Heterog Media 2:193

11. Carmi S, Cohen R, Dolev D (2006) Searching complex networks efficiently with minimal information. Europhys Lett 74:1102

12. Wu ZX, Wang WX, Yeung KH (2008) Traffic dynamics in scale-free networks with limited buffers and decongestion strategy. New J Phys 10:023025

13. Ashton DJ, Jarrett TC, Johnson NF (2005) Effect of congestion costs on shortest paths through complex networks. Phys Rev Lett 94:058701

14. Gourley S, Johnson NF (2006) Effects of decision-making on the transport costs across complex networks. Physica A 363:82

15. Pigou AC (1920) The economics of welfare. Macmillan, London

16. de Palma A, Lindsey R, Proost S (2006) Research challenges in modelling urban road pricing: an overview. Transp Pol 13:97

17. Helbing D, Schonhof M, Stark HU, Holyst JA (2005) How individuals learn to take turns: emergence of alternating cooperation in a congestion game and the prisoner's dilemma. Adv Complex Syst 8:87

18. Johansson O (1997) Optimal road-pricing: simultaneous treatment of time losses, increased fuel consumption, and emissions. Transp Res D 2:77

19. Rotaris L, Danielis R, Marcucci E, Massiani J (2010) The urban road pricing scheme to curb pollution in Milan, Italy: description, impacts and preliminary cost-benefit analysis assessment. Transp Res A 44:359

20. Zhang S, Campbell AM, Ehmke JF (2019) Impact of congestion pricing schemes on costs and emissions of commercial fleets in urban areas. Networks 73:466

21. Zheng ZD, Liu ZY, Liu CL, Shiwakoti N (2014) Understanding public response to a congestion charge: a random-effects ordered logit approach. Transp Res A 70:117

22. Sugiarto S, Miwa T, Sato H, Morikawa T (2017) Explaining differences in acceptance determinants toward congestion charging policies in Indonesia and Japan. J Urban Plann Dev 143:04016033

23. Liu ZY, Shiwakoti N, Bie YM (2018) Measuring the public acceptance of urban congestion-pricing: a survey in Melbourne (Australia). Transport 33:902

24. Link $\mathrm{H}$ (2015) Is car drivers' response to congestion charging schemes based on the correct perception of price signals? Transp Res A 71:96

Publisher's Note Springer Nature remains neutral with regard to jurisdictional claims in published maps and institutional affiliations. 